\title{
Sequence-Specific Association in Aqueous Media by Integrating Hydrogen Bonding and Dynamic Covalent Interactions
}

\author{
Minfeng Li, Kazuhiro Yamato, Joseph S. Ferguson and Bing Gong*
}

'Department of Chemistry

Natural Sciences Complex

University at Buffalo, The State University of New York

Buffalo, New York 14260

\section{Supporting Information}




\section{General}

All chemicals were purchased from Aldrich or Fisher (Acros) and were used as received unless otherwise indicated. The reactions were followed by thin-layer chromatography (precoated $0.25 \mathrm{~mm}$ silica gel plates from Aldrich), and silica gel column chromatography was carried out with silica gel 60 (mesh 230-400). ${ }^{1} \mathrm{H}$ NMR spectra were collected on at $400 \mathrm{MHz}$ and ${ }^{13} \mathrm{C}$ NMR spectra were recorded at $75 \mathrm{MHz}$ on Varian INOVA instruments. ${ }^{1} \mathrm{H}$ and ${ }^{13} \mathrm{C}$ NMR chemical shifts are reported as $\delta$ values in ppm relative to TMS or residual solvent. The ${ }^{1} \mathrm{H}$ chemical shifts $(\delta)$ were reported in parts per million downfield from TMS (tetramethylsilane). Carbon NMR spectra were recorded in ppm relative to the solvent signals: $\mathrm{CDCl}_{3}(77.23 \mathrm{ppm}),\left(\mathrm{CD}_{3}\right)_{2} \mathrm{SO}(39.52 \mathrm{ppm})$. Melting points were determined using a MEL-TEMP II apparatus. MALDI TOF MS spectra were recorded on a Bruker Bifrex IV MS spectrometer. 2,5-dihydroxybenzoic acid (DHB) or dithranol (DTH) were used as matrices. Electrospray ionization mass spectra (ESI-MS) were on a Themo Finnigan LCQ Advantage MS spectrometer.

\section{I-1 Common Procedures}

\section{(i) Coupling conditions}

To a stirred solution of acid compound (1 eq) and the amino compound (1 eq) in methylene chloride (DCM) (0.2 M) were added 1-ethyl-3-(3-dimethylaminopropyl) carbodiimide (EDCI) (1.2 eq), and N-hydroxybenzotriazole(HOBt) (1.2 eq) at $0^{\circ} \mathrm{C}$. Stirring was continued for $20 \mathrm{~h}$ while the mixture was warmed up to room temperature (RT). Solvent was evaporated, and the residue was dissolved in methylene cloride and extracted with $5 \%$ aqueous $\mathrm{HCl}$, saturated $\mathrm{NaHCO}_{3}$ solutions, and brine. The organic layer was dried over anhydrous $\mathrm{Na}_{2} \mathrm{SO}_{4}$ and concentrated in vacuo. Purification was accomplished by chromatography on silica gel by using $\mathrm{DCM} / \mathrm{MeOH}$ as eluent.

\section{(ii) Hydrolysis of the methyl esters}

Method a: The methyl ester was dissolved in methanol while cooling at RT. To this solutin, a solution of $\mathrm{NaOH}$ (1.5 eq) in water is added in several portions. The reaction was monitored by TLC. The resulting reaction mixture is concentrated in vacuo. The solution was acidified with $5 \% \mathrm{HCl}$ solution until $\mathrm{pH}=2$. Then $\mathrm{DCM}$ and brine were added and the two phases separated; the organic layer was dried over anhydrous $\mathrm{Na}_{2} \mathrm{SO}_{4}$ and evaporated in vacuo to yield the corresponding acid.

Method b: The methyl ester was dissolved in THF $(0.1 \mathrm{M})$ and cooled to $0^{\circ} \mathrm{C}$. A solution of $\mathrm{NaOH}(1 \mathrm{eq})$ in water was added in several portions. Stirring was continued at $0^{\circ} \mathrm{C}$ until TLC showed the consumption of all starting material. The solution was acidified with $5 \% \mathrm{HCl}$ solution until $\mathrm{pH}=1$. Then DCM was added, and the organic layer was separated, dried over anhydrous $\mathrm{Na}_{2} \mathrm{SO}_{4}$, and evaporated in vacuo to yield the corresponding acid. Purification was accomplished by chromatography on silica gel by using DCM/MeOH as eluent.

\section{(iii) Deprotection of t-butyloxycarbonyl (Boc)}

Protected compound was dissolved in anhydrous DCM (0.1 M) and TFA (80 eq) was added slowly at RT. Stirring was continued until TLC showed the consumption of all 
starting material. Then the mixture was concentrated in vacuo (EtOAc or $\mathrm{MeOH}$ was coevaporated several times to remove traces of TFA) to yield the TFA salt of the pure deprotected amino compound.

\section{(iv) Deprotection of benzyl esters}

A solution of the corresponding benzyl compound (1 eq) in $\mathrm{MeOH}$ and $\mathrm{Pd} / \mathrm{C}(10 \%)$ was shaken under a hydrogen atmosphere for 6 hours, then filtered through Celite. The filtrate was evaporated, yielding the corresponding acid.

\section{(v) Hydrogenantion of aromatic nitro group}

The nitro compound was dissolved in $\mathrm{MeOH}$ and $\mathrm{Pd} / \mathrm{C}(10 \%)$ was shaken under a hydrogen atmosphere for 6-10 hours, then filtered through Celite. The filtrate was evaporated, yielding the corresponding acid.

\section{I-2. Synthesis of strand 3:}
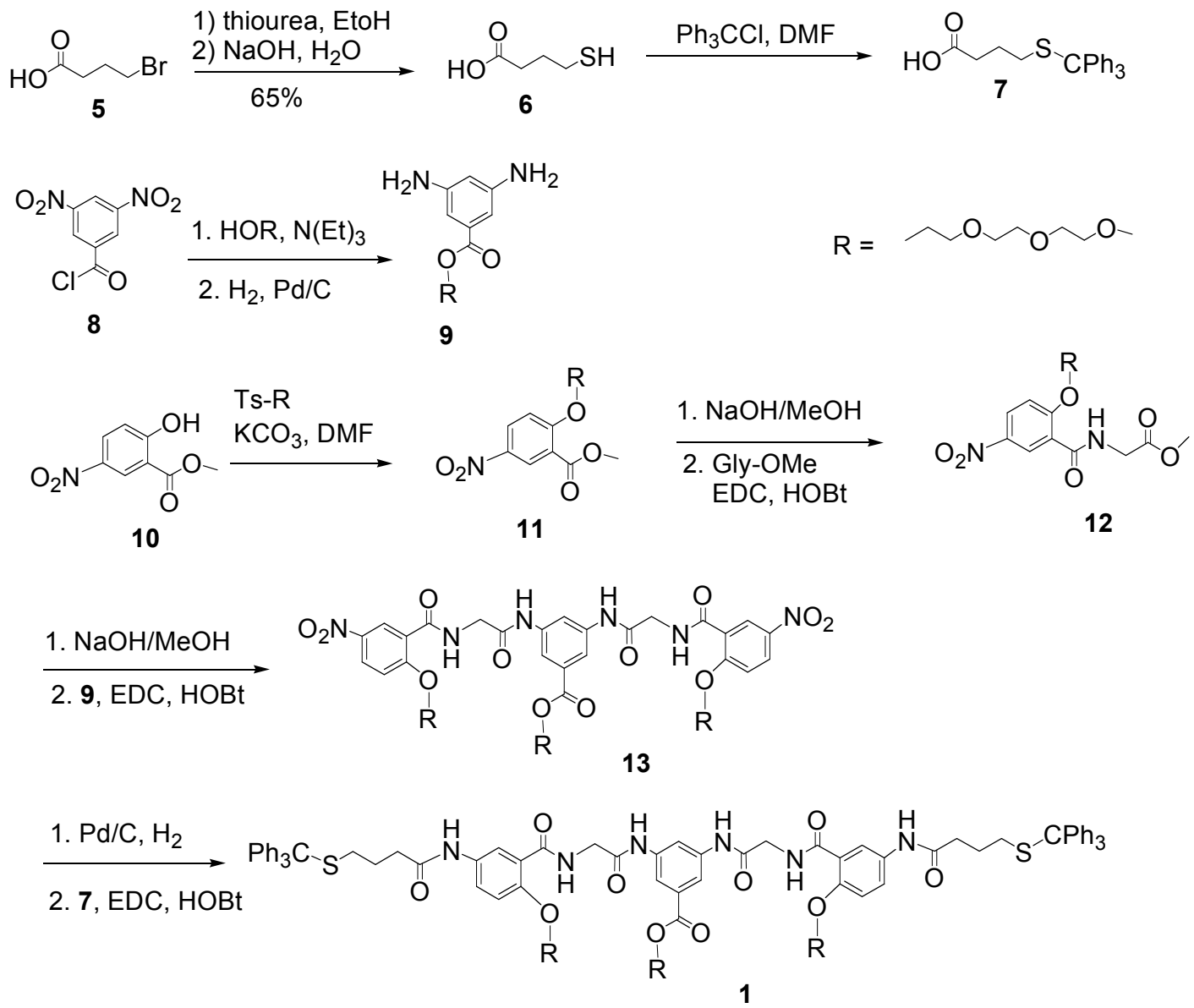

4-Mercapto-butyric acid (6). 4-Bromo-butyric acid (16.48 g, $98 \mathrm{mmol}$ ) was refluxed for $3 \mathrm{hr}$ with thiourea $(8.5 \mathrm{~g}, 107 \mathrm{mmol})$ in $30 \mathrm{ml}$ of ethanol. Then $25 \mathrm{ml}$ of aqueous $\mathrm{NaOH}$ (4 M, $100 \mathrm{mmol}$ )) was added and refluxing was continued for another $2 \mathrm{hr}$. The solution was concentrated in vacuo, extracted with ether (ether extract discarded), acidified with aqueous $\mathrm{HC} 1$ (6 M), and extracted with ether, the ethereal extract dried over anhydrous 
sodium sulfate, the ether evaporated, and the residue distilled in vacuo to give 6 (8.6 g, $63.7 \mathrm{mmol}, 65 \%)$ as a colorless oil. ${ }^{1} \mathrm{H} \mathrm{NMR}\left(\mathrm{CDCl}_{3}, 400 \mathrm{MHz}\right) \delta 2.56(\mathrm{~m}, 2 \mathrm{H}), \delta 2.23(\mathrm{t}$, $2 \mathrm{H}), \delta 1.86(\mathrm{~m}, 2 \mathrm{H}) ;{ }^{13} \mathrm{C} \mathrm{NMR}\left(300 \mathrm{MHz}, \mathrm{CDCl}_{3}\right) \delta 179.1,32.9,31.1,23.6$; ESI MS $\left[\mathrm{M}+\mathrm{Na}^{+}\right]$: expected, 153.02; observed, 153.0.

4-Tritylsulfanyl-butyric acid (7). Mixture of $4 \mathrm{~b}$ (3 g, $25 \mathrm{mmol})$ and trityl chloride (10 g, $38 \mathrm{mmol}$ ) were stirred in $40 \mathrm{~mL}$ DMF for 2 days at room temperature. A 10\% sodium acetate solution $(350 \mathrm{~mL})$ was then added, and the precipitate was filtered and washed with distilled water. Afterward, the residue was stirred in acetone at $50{ }^{\circ} \mathrm{C}$ for $30 \mathrm{~min}$ and filtered after cooling. The residue was washed with little acetone and diethyl ether. Recrystalization from $\mathrm{MeOH}$ afforded $7(5.4 \mathrm{~g}, 15 \mathrm{mmol}, 60 \%)$ as white powder. ${ }^{1} \mathrm{H}$ NMR $\left(\mathrm{CDCl}_{3}, 400 \mathrm{MHz}\right) \delta 7.11(\mathrm{~m}, 15 \mathrm{H}), \delta 2.41(\mathrm{t}, 2 \mathrm{H}), \delta 2.23(\mathrm{t}, 2 \mathrm{H}), \delta 1.90(\mathrm{~m}, 2 \mathrm{H})$; ${ }^{13} \mathrm{C} \mathrm{NMR}\left(300 \mathrm{MHz}, \mathrm{CDCl}_{3}\right) \delta 179.00,144.80,129.55,127.84,126.62,32.88,31.09$, 23.59; mp 130-131 ${ }^{\circ} \mathrm{C}$; ESI MS [M+Na ${ }^{+}$: expected, 385.13; observed, 385.1.

3,5-diamino-benzoic acid 2-[2-(2-methoxy-ethoxy)-ethoxy]-ethyl ester (9). A mixture of 2-[2-(2-Methoxy-ethoxy)-ethoxy]-ethanol $(20.5 \mathrm{~mL}, 0.13 \mathrm{~mol})$ and triethylamine (18 $\mathrm{mL}, 0.13 \mathrm{~mol}$ ) was dissolved in $200 \mathrm{~mL}$ of DCM and stirred at $0{ }^{0} \mathrm{C}$ for 10 minutes. To this mixture, a solution of 3,5-dinitro-benzoic chloride $(20 \mathrm{~g}, 87 \mathrm{mmol})$ in $40 \mathrm{~mL}$ of methylene chloride was added slowly. After addition, the reaction mixture was allowed to worm up to room temperature and stand at room temperature for 30 minutes, then washed with $1 \mathrm{M} \mathrm{HCl}$, saturated aqueous $\mathrm{NaHCO}_{3}$ and $\mathrm{NaCl}$ solution in sequence, dried over anhydrous $\mathrm{Na}_{2} \mathrm{SO}_{4}$, concentrated in vacuo to afforded the 3,5-dinitro benzoic acid 2-[2-(2-methoxy-ethoxy)-ethoxy]-ethyl ester. The dinitro groups were reduced by the general procedure $v$ to yield the corresponding diamine $9(25 \mathrm{~g}, 69.6 \mathrm{mmol}, 80 \%)$ as a brownish oil. ${ }^{1} \mathrm{H}$ NMR $(\mathrm{CDCl} 3,400 \mathrm{MHz}) \delta 6.60(\mathrm{~d}, 1 \mathrm{H}), \delta 5.71(\mathrm{~s}, 2 \mathrm{H}), \delta 4.42(\mathrm{~m}, 2 \mathrm{H})$, $\delta 3.81(\mathrm{~m}, 2 \mathrm{H}), \delta 3.67(\mathrm{~m}, 6 \mathrm{H}), \delta 3.55(\mathrm{~m}, 2 \mathrm{H}), \delta 3.38(\mathrm{~m}, 3 \mathrm{H}) ;{ }^{13} \mathrm{C}$ NMR $(300 \mathrm{MHz}$, $\left.\mathrm{CDCl}_{3}\right) \delta 166.80,147.52,131.91,106.88,105.64,71.87,70.64,70.55,70.51,69.19$, 63.89, 58.90; ESI MS [M+Na $]$ : expected, 298.15; observed, 321.1.

\section{2-\{2-[2-(2-methoxy-ethoxy)-ethoxy]-ethoxy\}-5-nitro-benzoic acid methyl ester (11).}

2-Hydroxy-5-nitro-benzoic acid methyl ester $\mathbf{6}^{1}(26 \mathrm{~g}, 0.15 \mathrm{~mol})$ and toluene-4-sulfonic acid 2-[2-(2-methoxy-ethoxy)-ethoxy]-ethyl ester $(53 \mathrm{~g}, 0.15 \mathrm{~mol})$ were dissolved in 250 $\mathrm{mL} \mathrm{N}, \mathrm{N}$-dimethyl formamide follwed by addition of potassium carbonate $(21 \mathrm{~g}, 0.15$ mol). The resulting mixture was stirred at $80{ }^{\circ} \mathrm{C}$ for 10 hours. The reaction mixture was then filtered, concentrated in vacuo, poured into brine, extracted three time with ethyl acetate. The orginc phase was dried over anhydrous sodium sulfate, filtered and concentrated in vacuo. Purification was accomplished by chromatography on silica gel by using DCM/MeOH as eluent to give 11, $39 \mathrm{~g}$ as a light yellow oil ( $90 \%$ yield). ${ }^{1} \mathrm{H} \mathrm{NMR}$ $\left(\mathrm{CDCl}_{3}, 400 \mathrm{MHz}\right) 7.10(\mathrm{~d}, 1 \mathrm{H}), 8.34(\mathrm{dd}, 9.2,1 \mathrm{H}), 8.80(\mathrm{~d}, 1 \mathrm{H}), \delta 4.42(\mathrm{~m}, 2 \mathrm{H}), 4.05(\mathrm{~s}$, $3 \mathrm{H}), \delta 3.81(\mathrm{~m}, 2 \mathrm{H}), \delta 3.67(\mathrm{~m}, 6 \mathrm{H}), \delta 3.55(\mathrm{~m}, 2 \mathrm{H}), \delta 3.38(\mathrm{~m}, 3 \mathrm{H}) ;{ }^{13} \mathrm{C}$ NMR $(300$ $\left.\mathrm{MHz}, \mathrm{CDCl}_{3}\right) \delta 164.96,163.50,141.23,129.22,128.22,121.32,113.85,72.41,71.57$, 71.15, 71.01, 70.08, 69.75, 59.48, 52.92; ESI MS [M+Na $\left.{ }^{+}\right]$: expected, 366.13; observed, 366.1 . 

methyl ester (12). 7 (4 g, $12 \mathrm{mmol}$ ) was hydrolyzed by the method a to give the corresponding acid which was used without further purification to couple with Gly-OMe hydrochloric salt $(2.3 \mathrm{~g}, 18 \mathrm{mmol})$ following the general procedure $i$. Purification was accomplished by chromatography on silica gel by using DCM/MeOH as eluent to afford $12(3.4 \mathrm{~g}, 8.4 \mathrm{mmol}, 70 \%){ }^{1} \mathrm{H}$ NMR $\left(\mathrm{CDCl}_{3}, 400 \mathrm{MHz}\right) \delta 8.62(\mathrm{~d}, 1 \mathrm{H}), \delta 8.58(\mathrm{t}, 1 \mathrm{H}), \delta$ $8.36(\mathrm{~d}, 1 \mathrm{H}), \delta 7.44(\mathrm{~d}, 1 \mathrm{H}), \delta 4.43(\mathrm{t}, 2 \mathrm{H}), \delta 4.02(\mathrm{t}, 2 \mathrm{H}), \delta 3.86(\mathrm{t}, 2 \mathrm{H}), \delta 3.65(\mathrm{~m}, 2 \mathrm{H})$, $\delta 3.61(\mathrm{~m}, 2 \mathrm{H}), \delta 3.52(\mathrm{~m}, 2 \mathrm{H}), \delta 3.36(\mathrm{~s}, 3 \mathrm{H}) ;{ }^{13} \mathrm{C} \mathrm{NMR}\left(300 \mathrm{MHz}, \mathrm{CDCl}_{3}\right) \delta 169.0$, $164.96,163.50,141.23,129.22,128.22,121.32,113.85,72.41,71.57,71.15,71.01$, 70.08, 69.75, 59.48, 52.92, 48.61;ESI MS [M+Na $\left.{ }^{+}\right]$: expected, 423.15; observed, 423.2.

\section{3,5-Bis-[2-(2-\{2-[2-(2-methoxy-ethoxy)-ethoxy]-ethoxy\}-5-nitro-benzoylamino)-} acetylamino]-benzoic acid 2-[2-(2-methoxy-ethoxy)-ethoxy]-ethyl ester (13). 8 (622 $\mathrm{mg}, 1.6 \mathrm{mmol}$ ) was hydrolyzed by the method a to give the corresponding acid which was used without further purification to couple with diamine 5 (200 $\mathrm{mg}, 0.67 \mathrm{mmol})$ following the general procedure $i$. Purification was accomplished by chromatography on silica gel by using DCM/MeOH as eluent to afford $13(520 \mathrm{mg}, 0.5 \mathrm{mmol}, 75 \%)$ as a thick brown oil that slowly solidifies. ${ }^{1} \mathrm{H}$ NMR $\left(\mathrm{CDCl}_{3}, 400 \mathrm{MHz}\right) \delta 10.23(\mathrm{~s}, 2 \mathrm{H}), \delta$ $8.943(\mathrm{~d}, 2 \mathrm{H}, \mathrm{J}=2.7 \mathrm{~Hz}), \delta 8.66(\mathrm{t}, 2 \mathrm{H}), \delta 8.39(\mathrm{dd}, 2 \mathrm{H}, \mathrm{J}=2.7,9.0 \mathrm{~Hz}), \delta 8.31(\mathrm{~s}, 1 \mathrm{H}), \delta$ $7.27(\mathrm{~d}, 2 \mathrm{H}, \mathrm{J}=9.1 \mathrm{~Hz}), \delta 4.44(\mathrm{~m}, 2 \mathrm{H}), \delta 4.31(\mathrm{~d}, 4 \mathrm{H}, \mathrm{J}=5.1 \mathrm{~Hz}), \delta 3.82(\mathrm{~m}, 2 \mathrm{H}), \delta$ $3.68(\mathrm{~m}, 6 \mathrm{H}), \delta 3.51(\mathrm{~m}, 2 \mathrm{H}), \delta 3.32(\mathrm{~m}, 4 \mathrm{H}), \delta 2.58(\mathrm{~m}, 6 \mathrm{H}) ;{ }^{13} \mathrm{C} \mathrm{NMR}(300 \mathrm{MHz}$, $\left.\mathrm{CDCl}_{3}\right) \delta 166.20,166.80,164.96,163.50,147.52,141.23,131.91,129.22,128.22$, $121.32,113.85,106.88,105.64,72.41,71.57,71.15,71.01,70.08,69.75,59.48,48.61$; ESI MS [M+Na $\left.{ }^{+}\right]$: expected, 1057.40; observed, 1057.4.

\section{3,5-Bis-\{2-[2-\{2-[2-(2-methoxy-ethoxy)-ethoxy]-ethoxy\}-5-(4-tritylsulfanyl- butyrylamino)-benzoylamino]-acetylamino\}-benzoic acid 2-[2-(2-methoxy-ethoxy)- ethoxy]-ethyl ester (1). Dinitro compound 9 was quantitatively reduced to corresponding diamine $(100 \mathrm{mg}, 100 \mu \mathrm{mol})$ which was coupled with the acid $3(112 \mathrm{mg}, 300 \mu \mathrm{mol})$ following the general procedure $i$ Purification was accomplished by chromatography on silica gel by using DCM/MeOH as eluent and further purified by reverse phase HPLC to afforded $10(133 \mathrm{mg}, 80 \mu \mathrm{mol}, 80 \%)$ as a white gel. ${ }^{1} \mathrm{H}$ NMR $\left(\mathrm{CDCl}_{3}, 400 \mathrm{MHz}\right) \delta 10.23$ $(\mathrm{s}, 2 \mathrm{H}), \delta 8.943(\mathrm{~d}, 2 \mathrm{H}, \mathrm{J}=2.7 \mathrm{~Hz}), \delta 8.66(\mathrm{t}, 2 \mathrm{H}), \delta 8.39(\mathrm{dd}, 2 \mathrm{H}, \mathrm{J}=2.7,9.0 \mathrm{~Hz}), \delta 8.31$ $(\mathrm{s}, 1 \mathrm{H}), \delta 7.27(\mathrm{~d}, 2 \mathrm{H}, \mathrm{J}=9.1 \mathrm{~Hz}), \delta 7.11(\mathrm{~m}, 15 \mathrm{H}), \delta 4.44(\mathrm{~m}, 2 \mathrm{H}), \delta 4.31(\mathrm{~d}, 4 \mathrm{H}, \mathrm{J}=5.1$ $\mathrm{Hz}), \delta 3.82(\mathrm{~m}, 2 \mathrm{H}), \delta 3.68(\mathrm{~m}, 6 \mathrm{H}), \delta 3.51(\mathrm{~m}, 2 \mathrm{H}), \delta 3.32(\mathrm{~m}, 4 \mathrm{H}), \delta 2.58(\mathrm{~m}, 6 \mathrm{H}), \delta$ $2.41(\mathrm{t}, 2 \mathrm{H}), \delta 2.23(\mathrm{t}, 2 \mathrm{H}) . \delta 1.90(\mathrm{~m}, 2 \mathrm{H}) ;{ }^{13} \mathrm{C} \mathrm{NMR}\left(300 \mathrm{MHz}, \mathrm{CDCl}_{3}\right) \delta$ 179.10, $166.20,166.80,164.96,163.50,147.52,141.23,131.91,129.22,128.22,121.32,113.85$, 106.88, 105.64, 72.41, 71.57, 71.15, 71.01, 70.08, 69.75, 59.48, 48.61, 32.9, 31.1, 23.6; ESI MS $\left[\mathrm{M}+\mathrm{Na}^{+}\right]$: expected, $1685.7 ;$ observed, 1685.7.}




\section{I-3. Synthesis of strand 2}
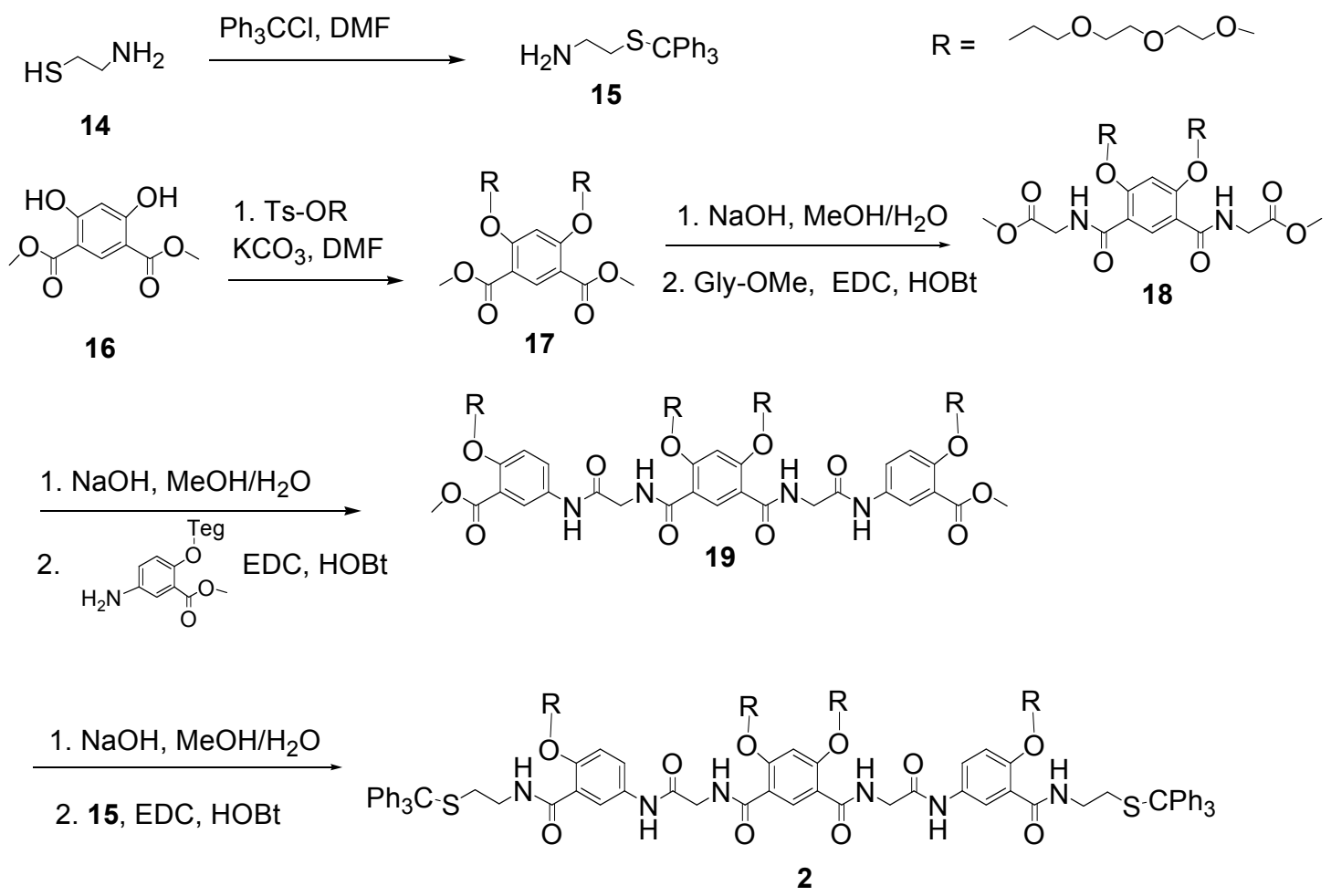

2-Tritylsulfanyl-ethylamine (15). The procedures was the same as that of preparation of compound 7. Recrystailization from ethyl acetate/hexanes to afford 15 (77\%) as a brown oil. ${ }^{1} \mathrm{H}$ NMR $\left(\mathrm{CDCl}_{3}, 400 \mathrm{MHz}\right) \delta 7.01(\mathrm{~m}, 15 \mathrm{H}), \delta 3.02(\mathrm{t}, 2 \mathrm{H}), \delta 2.70(\mathrm{t}, 2 \mathrm{H}) ;{ }^{13} \mathrm{C}$ NMR $\left(300 \mathrm{MHz}, \mathrm{CDCl}_{3}\right) \delta 144.90,129.59,127.84,126.64,66.57,41.01,36.21$; ESI MS $\left[\mathrm{M}+\mathrm{Na}^{+}\right]$: expected, 342.14; observed, 342.1.

4,6-bis-\{2-[2-(2-methoxy-ethoxy)-ethoxy]-ethoxy\}-isophthalic acid dimethylester (17). 4,6-Dihydroxy-isophthalic acid dimethyl ester $16^{1}$ was alkylated with toluene-4-sulfonic acid 2-[2-(2-methoxy-ethoxy)-ethoxy]-ethyl ester following the same procedure of the preparation of 7 to afford 17 as a redish oil (70\%). $\left.{ }^{1} \mathrm{H} \mathrm{NMR} \mathrm{(CDCl3,} 400 \mathrm{MHz}\right), \delta 8.78$ (s, $1 \mathrm{H}), \delta 6.62(\mathrm{~s}, 1 \mathrm{H}), \delta 4.38(\mathrm{bt}, 4 \mathrm{H}), \delta 3.95$ (bt, 4H), $\delta 3.76(\mathrm{~m}, 4 \mathrm{H}), \delta 3.69(\mathrm{~m}, 4 \mathrm{H})$,

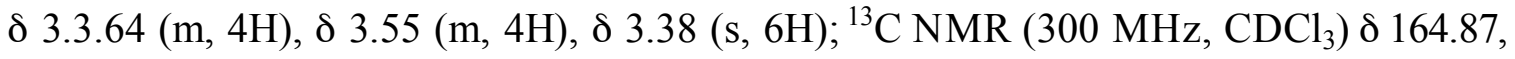
162.18, 139.05, 112,81, 98.35, 71.81, 70.70, 70.41, 70.34, 69.50, 68.62, 58.86, 53.05; ESI MS [M+Na $\left.{ }^{+}\right]$: expected, 342.14; observed, 342.1.

(5-(Methoxycarbonylmethyl-carbamoyl)-2,4-bis-\{2-[2-(2-methoxy-ethoxy)-ethoxy]ethoxy\}-benzoylamino)-acetic acid methyl ester (18). 17 (1.04 g, 2 mmol) was hydrolyzed by the method a to give the corresponding acid which was used without further purification to couple with Gly-OMe $(0.5 \mathrm{~g}, 0.4 \mathrm{mmol})$ following the general procedure $i$. Purification was accomplished by chromatography on silica gel by using $\mathrm{DCM} / \mathrm{MeOH}$ as eluent to afford $18(0.95 \mathrm{~g}, 1.5 \mathrm{mmol}, 75 \%)$ as a thick brown oil. ${ }^{1} \mathrm{H}$ NMR (CDCl3, $400 \mathrm{MHz}) \delta 8.98(\mathrm{~s}, 1 \mathrm{H}), \delta 8.32(\mathrm{t}, 3 \mathrm{H}), \delta 6.52(\mathrm{~s}, 1 \mathrm{H}), \delta 4.31(\mathrm{bt}, 4 \mathrm{H}), \delta$ $4.26(\mathrm{~d}, 2 \mathrm{H}), \delta 3.95(\mathrm{bt}, 4 \mathrm{H}), \delta 3.76(\mathrm{~m}, 4 \mathrm{H}), \delta 3.69(\mathrm{~m}, 4 \mathrm{H}), \delta 3.3 .64(\mathrm{~m}, 4 \mathrm{H}), \delta 3.55$ (m, 
$4 \mathrm{H}), \delta 3.38(\mathrm{~s}, 6 \mathrm{H}) ;{ }^{13} \mathrm{C}$ NMR $\left(300 \mathrm{MHz}, \mathrm{CDCl}_{3}\right) \delta 179.01,164.87,162.18,139.05$, $112,81,98.35,71.81,70.70,70.41,70.34,69.50,68.62,58.86,53.05,48.55$; ESI MS $\left[\mathrm{M}+\mathrm{Na}^{+}\right]$: expected, 655.28; observed, 655.3.

5-\{2-[2,4-Bis-\{2-[2-(2-methoxy-ethoxy)-ethoxy]-ethoxy $\}-5-($ methylcarbamoylmethylcarbamoyl)-benzoylamino]-\{5-[2-[2-(2-methoxy-ethoxy)-ethoxy]-benzoyl]-2-\{2-[2-(2methoxy-ethoxy)-ethoxy]-ethoxy\}-benzoic acid dimethyl ester (19). 15 (0.95 g, 1.5 mmol) was hydrolyzed by the method a to give the corresponding acid which was used without further purification to couple with hydrogenation product of 7 (procedure $\boldsymbol{v}$, without further purification) $(0.95 \mathrm{~g}, 0.3 \mathrm{mmol})$ following the general procedure $i$. Purification was accomplished by chromatography on silica gel by using DCM/MeOH as eluent to afford 19 (1.3 g, $1.1 \mathrm{mmol}, 70 \%) .{ }^{1} \mathrm{H} \mathrm{NMR}\left(\mathrm{CDCl}_{3}, 400 \mathrm{MHz}\right) \delta 9.47(\mathrm{~s}, 2 \mathrm{H}), \delta$ $8.72(\mathrm{~d}, 1 \mathrm{H}, \delta 8.61(\mathrm{~s}, 2 \mathrm{H}), \delta 8.28(\mathrm{~s}, 2 \mathrm{H}),, \delta 8.00(\mathrm{~d}, 2 \mathrm{H}, \mathrm{J}=7.8 \mathrm{~Hz}), \delta 7.03(\mathrm{~d}, 2 \mathrm{H}, \mathrm{J}=$ $8.0 \mathrm{~Hz}), \delta 6.50(\mathrm{~s}, 1 \mathrm{H}), \delta 4.44(\mathrm{~m}, 2 \mathrm{H}), \delta 4.31(\mathrm{~d}, 4 \mathrm{H}, \mathrm{J}=5.1 \mathrm{~Hz}), \delta 3.82(\mathrm{~m}, 2 \mathrm{H}), \delta 3.68$ $(\mathrm{m}, 9 \mathrm{H}), \delta 3.51(\mathrm{~m}, 8 \mathrm{H}), \delta 3.32(\mathrm{~m}, 4 \mathrm{H}), \delta 3.37(\mathrm{~s}, 6 \mathrm{H}), \delta 3.28(\mathrm{~s}, 6 \mathrm{H}) ;{ }^{13} \mathrm{C} \mathrm{NMR}(300$ $\left.\mathrm{MHz}_{,} \mathrm{CDCl}_{3}\right) \delta 181.57,164.96,164.87,163.50,162.18,141.23,139.05,129.22,128.22$ $112.81,121.32,113.85,98.35,72.41,71.57,71.81,71.15,71.01,70.70,70.41,70.34$, $70.08,69.50,69.75,68.62,59.92,58.86,53.05$; ESI MS $\left[\mathrm{M}+\mathrm{Na}^{+}\right]$: expected 1217.53; observed, 1217.5 .

\section{4,6-Bis-\{2-[2-(2-methoxy-ethoxy)-ethoxy]-ethoxy\}-N,N'-bis-\{[4-\{2-[2-(2-methoxy- ethoxy)-ethoxy]-ethoxy\}-3-(2-tritylsulfanyl-ethylcarbamoyl)-phenylcarbamoyl]-} methyl\}-isophthalamide (2). $19(0.24 \mathrm{~g}, 0.2 \mathrm{mmol})$ was hydrolyzed by the method a to give the corresponding acid which was used without further purification to couple with $12(0.13 \mathrm{~g}, 0.4 \mathrm{mmol})$ following the general procedure $i$. Purification was accomplished by chromatography on silica gel by using $\mathrm{DCM} / \mathrm{MeOH}$ as eluent and further purified by reverse phase HPLC to afford $16(0.15 \mathrm{~g}, 0.13 \mathrm{mmol}, 65 \%)$ as a white gel. ${ }^{1} \mathrm{H} \mathrm{NMR}$ $\left(\mathrm{CDCl}_{3}, 400 \mathrm{MHz}\right) \delta 9.47(\mathrm{~s}, 2 \mathrm{H}), \delta 8.72(\mathrm{~d}, 1 \mathrm{H}, \delta 8.61(\mathrm{~s}, 2 \mathrm{H}), \delta 8.28(\mathrm{~s}, 2 \mathrm{H}),, \delta 8.00$ $(\mathrm{d}, 2 \mathrm{H}, \mathrm{J}=7.8 \mathrm{~Hz}), \delta 8.05(\mathrm{t}, 1 \mathrm{H}), \delta 7.03(\mathrm{~d}, 2 \mathrm{H}, \mathrm{J}=8.0 \mathrm{~Hz}), \delta 7.01(\mathrm{~m}, 15 \mathrm{H}), \delta 6.50$ (s, $1 \mathrm{H}), \delta 4.44(\mathrm{~m}, 2 \mathrm{H}), \delta 4.31(\mathrm{~d}, 4 \mathrm{H}, \mathrm{J}=5.1 \mathrm{~Hz}), \delta 4.35(\mathrm{~d}, 2 \mathrm{H}), \delta 3.82(\mathrm{~m}, 2 \mathrm{H}), \delta 3.68$ $(\mathrm{m}, 9 \mathrm{H}), \delta 3.51(\mathrm{~m}, 8 \mathrm{H}), \delta 3.32(\mathrm{~m}, 4 \mathrm{H}), \delta 3.37(\mathrm{~s}, 6 \mathrm{H}), \delta 3.28(\mathrm{~s}, 6 \mathrm{H})$, , $\delta 3.02(\mathrm{t}, 2 \mathrm{H})$, $\delta 2.70(\mathrm{t}, 2 \mathrm{H}), \delta 2.02(\mathrm{~s}, 3 \mathrm{H}) ;{ }^{13} \mathrm{C} \mathrm{NMR}\left(300 \mathrm{MHz}, \mathrm{CDCl}_{3}\right) \delta 181.57,164.96,164.87$, $163.50,162.18,144.90,141.23,139.05,129.59,129.22,128.22,127.84,126.64,112.81$, $121.32,113.85,98.35,72.41,71.57,71.81,71.15,71.01,70.70,70.41,70.34,70.08$, $69.50,69.75,68.62,66.57,59.92,58.86,53.05,41.01,36.21 ;$ ESI MS [M+Na $\left.{ }^{+}\right]$:

expected 1791.76; observed, 1791.7. 


\section{I-4. Synthesis of strand 3}

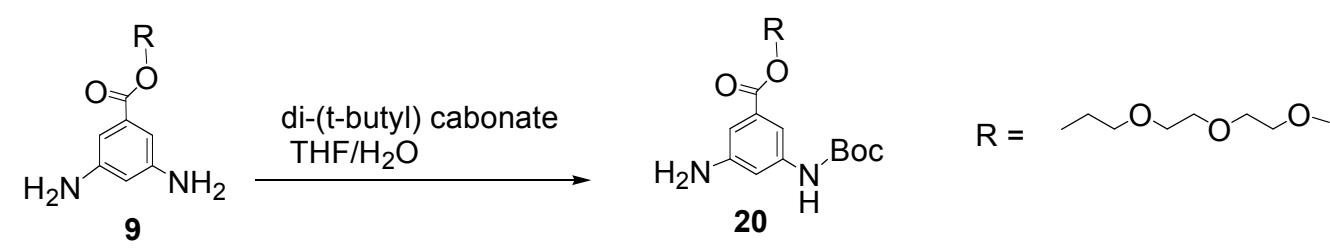

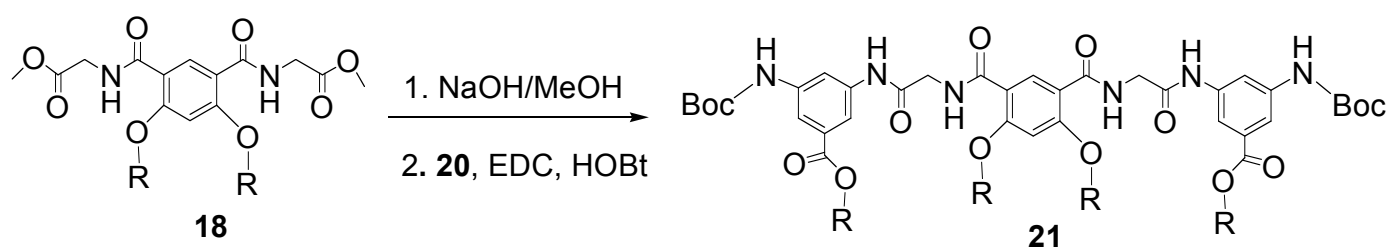

1. TFA/DCM 2. 3, EDC, $\mathrm{HOBt}$

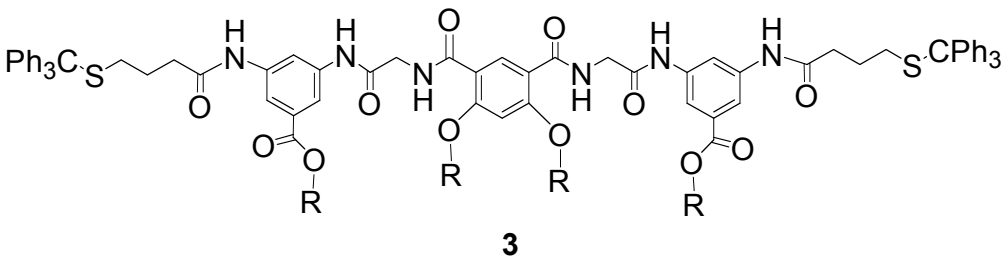

3-Amino-5-tert-butoxycarbonylamino-benzoic acid 2-[2-(2-methoxy-ethoxy)ethoxy]-ethyl ester (20). Diamine 9, (6 g, $20 \mathrm{mmol})$ and di-(tert-butyl) carbonate (4.3 $\mathrm{g}$, $20 \mathrm{mmol}$ ) were slurried in $200 \mathrm{~mL}$ of THF at $0{ }^{\circ} \mathrm{C}$. To this mixture, $1 \mathrm{M} \mathrm{NaOH}$ aqueous solution, $22 \mathrm{~mL}$, was added, over 30 minutes. The reaction mixture was stirred overnight and allowed to warm to room temperature. To the reaction mixture was added ethyl acetate and brine. The resulting layers were separated and the aqueous layer was again extracted with ethyl acetate. The combined organics were washed with brine, dried with anhydrous sodium sulfate, filtered, and evaporated. Purification was accomplished by chromatography on silica gel by using $\mathrm{DCM} / \mathrm{MeOH}$ as eluent to afford 18 (4.3 g 11 mmol, 55\%) as a yellowish oil. ${ }^{1} \mathrm{H}$ NMR $(\mathrm{CDCl} 3,400 \mathrm{MHz}) \delta 8.06(\mathrm{t}, 1 \mathrm{H}), \delta 7.71(\mathrm{~s}, 1 \mathrm{H})$, $\delta 7.04(\mathrm{~s}, 1 \mathrm{H}), \delta 6.94(\mathrm{~s}, 1 \mathrm{H}) \delta 4.42(\mathrm{~m}, 2 \mathrm{H}), \delta 3.81(\mathrm{~m}, 2 \mathrm{H}), \delta 3.67(\mathrm{~m}, 6 \mathrm{H}), \delta 3.55(\mathrm{~m}$, $2 \mathrm{H}), \delta 3.38(\mathrm{~m}, 3 \mathrm{H}), \delta$ 1,51 (s, 9H). ${ }^{13} \mathrm{C} \mathrm{NMR}\left(300 \mathrm{MHz}, \mathrm{CDCl}_{3}\right) \delta 166.38,152.67$, 147.42 , 139.58, 131.47, 110.54, 109.68, 109.06, 71.93, 70.75, 70.60, 70.57, 69.16, 64.11, 58.97, 28.30; ESI MS [M+Na ${ }^{+}$: expected 421.21; observed, 421.2.

4,6-Bis-\{2-[2-(2-methoxy-ethoxy)-ethoxy]-ethoxy\}-N,N'-3-[2-(2,4-Bis-\{2-[2-(2methoxy-ethoxy)-ethoxy]-ethoxy\}-5-methylcarbamoyl-benzoylamino)]-5-tertbutoxycarbonylamino-l-isophthalamide (21). $18(0.95 \mathrm{~g}, 1 \mathrm{mmol})$ was hydrolyzed by the method a to give the corresponding acid which was used without further purification to couple with 18 following the general procedure $(i)$. Purification was accomplished by chromatography on silica gel by using $\mathrm{DCM} / \mathrm{MeOH}$ as eluent to afford $19(1.3 \mathrm{~g}, 1.1$ mmol, 70\%) as a thick brown oil. ${ }^{1} \mathrm{H} \mathrm{NMR}\left(\mathrm{CDCl}_{3}, 400 \mathrm{MHz}\right) \delta 9.47(\mathrm{~s}, 2 \mathrm{H}), \delta 8.72(\mathrm{~d}$, $1 \mathrm{H}, \delta 8.61(\mathrm{~s}, 2 \mathrm{H}), \delta 8.28(\mathrm{~s}, 2 \mathrm{H}),, \delta 8.00(\mathrm{~d}, 2 \mathrm{H}, \mathrm{J}=7.8 \mathrm{~Hz}), \delta 8.05(\mathrm{t}, 1 \mathrm{H}), \delta 7.03(\mathrm{~d}, 2 \mathrm{H}$, $\mathrm{J}=8.0 \mathrm{~Hz}), \delta 7.01(\mathrm{~m}, 15 \mathrm{H}), \delta 6.50(\mathrm{~s}, 1 \mathrm{H}), \delta 4.44(\mathrm{~m}, 2 \mathrm{H}), \delta 4.31(\mathrm{~d}, 4 \mathrm{H}, \mathrm{J}=5.1 \mathrm{~Hz}), \delta$ $4.35(\mathrm{~d}, 2 \mathrm{H}), \delta 3.82(\mathrm{~m}, 2 \mathrm{H}), \delta 3.68(\mathrm{~m}, 9 \mathrm{H}), \delta 3.51(\mathrm{~m}, 8 \mathrm{H}), \delta 3.32(\mathrm{~m}, 4 \mathrm{H}), \delta 3.37(\mathrm{~s}$, 
$6 \mathrm{H}), \delta 3.28(\mathrm{~s}, 6 \mathrm{H})$, , $\delta 3.02(\mathrm{t}, 2 \mathrm{H}), \delta 2.70(\mathrm{t}, 2 \mathrm{H}), \delta 2.02(\mathrm{~s}, 3 \mathrm{H}) .{ }^{13} \mathrm{C} \mathrm{NMR}(300 \mathrm{MHz}$, $\left.\mathrm{CDCl}_{3}\right) \delta 179.01,166.38,164.87,162.18,152.67,147.42,139.58,139.05,131.47$, $112,81,110.54,109.68,109.06,98.35,71.81,71.93,70.75,70.70,70.60,70.57,70.41$, $70.34,69.50,69.16,68.62,64.11,58.97,58.86,53.05,28.31$; ESI MS $\left[\mathrm{M}+\mathrm{Na}^{+}\right]$: expected 1387.64; observed, 1387.7.

\section{4,6-Bis-\{2-[2-(2-methoxy-ethoxy)-ethoxy]-ethoxy\}-N,N'-bis-\{3-amino-5-(2-[2-(2-}

methoxy-ethoxy)-ethoxy]-ethoxy-ethylcarbamoyl)\}-phenylamino\}\}-3-(2tritylsulfanyl-ethylcarbamoyl)-phenylcarbamoyl]-methyl\}-isophthalamide (3).

The BOC groups of $20(0.14 \mathrm{~g}, 0.1 \mathrm{mmol})$ were removed following general procedure (iii) to give the TFA salt of the corresponding amine, which was coupled with $\mathbf{3}(72.5 \mathrm{mg}$, $2 \mathrm{mmol}$ ) following general procedure (i). Purification was accomplished by chromatography on silica gel by using $\mathrm{DCM} / \mathrm{MeOH}$ as eluent and further purified by reverse phase HPLC to afford $19(0.12 \mathrm{~g}, 0.065 \mathrm{mmol}, 65 \%)$ as a white gel. ${ }^{1} \mathrm{H} \mathrm{NMR}$ $\left(\mathrm{CDCl}_{3}, 400 \mathrm{MHz}\right) \delta 9.47(\mathrm{~s}, 2 \mathrm{H}), \delta 8.72(\mathrm{~d}, 1 \mathrm{H}, \delta 8.61(\mathrm{~s}, 2 \mathrm{H}), \delta 8.28(\mathrm{~s}, 2 \mathrm{H}),, \delta 8.00(\mathrm{~d}$, $2 \mathrm{H}, \mathrm{J}=7.8 \mathrm{~Hz}), \delta 8.05(\mathrm{t}, 1 \mathrm{H}), \delta 7.03(\mathrm{~d}, 2 \mathrm{H}, \mathrm{J}=8.0 \mathrm{~Hz}), \delta 7.01(\mathrm{~m}, 15 \mathrm{H}), \delta 6.50(\mathrm{~s}, 1 \mathrm{H})$, $\delta 4.44(\mathrm{~m}, 2 \mathrm{H}), \delta 4.31(\mathrm{~d}, 4 \mathrm{H}, \mathrm{J}=5.1 \mathrm{~Hz}), \delta 4.35(\mathrm{~d}, 2 \mathrm{H}), \delta 3.82(\mathrm{~m}, 2 \mathrm{H}), \delta 3.68(\mathrm{~m}, 9 \mathrm{H})$, $\delta 3.51(\mathrm{~m}, 8 \mathrm{H}), \delta 3.32(\mathrm{~m}, 4 \mathrm{H}), \delta 3.37(\mathrm{~s}, 6 \mathrm{H}), \delta 3.28(\mathrm{~s}, 6 \mathrm{H}), \delta 3.02(\mathrm{t}, 2 \mathrm{H}), \delta 2.70(\mathrm{t}$, $2 \mathrm{H}), \delta 2.02(\mathrm{~s}, 3 \mathrm{H}) .{ }^{13} \mathrm{C}$ NMR $\left(300 \mathrm{MHz} \mathrm{CDCl}_{3}\right) \delta 179.01,166.38,164.87,162.18$, $152.67,159.15,144.80,147.42,139.58,139.05,131.47,129.55,127.84,126.62,112,81$, $110.54,109.68,109.06,98.35,71.81,71.93,70.75,70.70,70.60,70.57,70.41,70.34$, $69.50,69.16,68.62$, , 64.11, 58.97,58.86, 53.05, 32.88, 31.09, 23.59; ESI MS [M+Na $\left.{ }^{+}\right]$: expected 1875.78; observed, 1875.8 .

\section{I-5. Synthesis of strand 4}

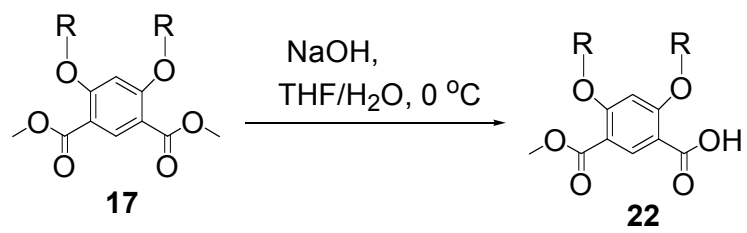

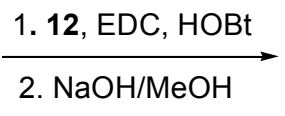

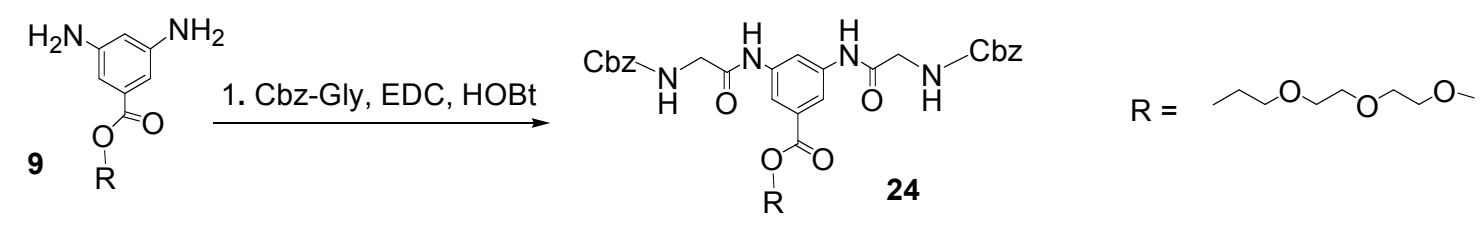

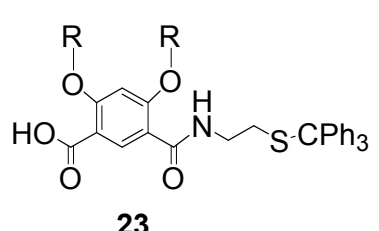

23

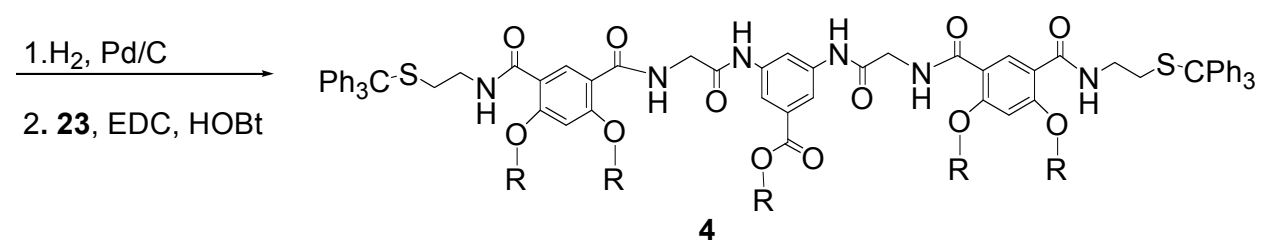

4,6-Bis-\{2-[2-(2-methoxy-ethoxy)-ethoxy]-ethoxy\}-isophthalic acid monomethyl ester (22). 14 (5.2 g, $10 \mathrm{mmol})$ was hydrolyzed by the method a to give the mono acid 21 (3.5 g, $7 \mathrm{mmol}) .{ }^{1} \mathrm{H} \mathrm{NMR}\left(\mathrm{CDCl}_{3}, 400 \mathrm{MHz}\right) \delta 8.88(\mathrm{~s}, 1 \mathrm{H}), \delta 6.57(\mathrm{~s}, 1 \mathrm{H}), \delta 4.36(\mathrm{t}, 2 \mathrm{H}), \delta$ $4.28(\mathrm{t}, 2 \mathrm{H}),, \delta 3.93(\mathrm{t}, 2 \mathrm{H}),, \delta 3.88(\mathrm{~s}, 3 \mathrm{H}), \delta 3.86(\mathrm{t}, 2 \mathrm{H}), \delta 3,74(\mathrm{~m}, 2 \mathrm{H}),, \delta 3.68(\mathrm{~m}$, 
$2 \mathrm{H}), \delta 3.56(\mathrm{~m}, 6 \mathrm{H}), \delta 3.48(\mathrm{~m}, 2 \mathrm{H}), \delta 3.37$ (s, 3H), $\delta 3.34(\mathrm{~s}, 3 \mathrm{H}) ;{ }^{13} \mathrm{C} \mathrm{NMR}(300 \mathrm{MHz}$, $\left.\mathrm{CDCl}_{3}\right) \delta 164.87,162.18,139.05,112.81,98.35,71.81,70.70,70.41,70.34,69.50,68.62$, 58.86; ESI MS [M+Na ${ }^{+}$: expected 527.22; observed, 527.2.

4,6-Bis-\{2-[2-(2-methoxy-ethoxy)-ethoxy]-ethoxy\}-N-(2-tritylsulfanyl-ethyl)isophthalamic acid (23). $21(2.5 \mathrm{~g}, 5 \mathrm{mmol})$ was coupled with $12(1.6 \mathrm{~g}, 5 \mathrm{mmol})$ following general procedure (i) to afford $22(2.9 \mathrm{~g}, 3.75 \mathrm{mmol}, 75 \%) .{ }^{1} \mathrm{H} \mathrm{NMR}\left(\mathrm{CDCl}_{3}\right.$, $400 \mathrm{MHz}) \delta 8.88(\mathrm{~s}, 1 \mathrm{H}), \delta 3.37(\mathrm{~s}, 3 \mathrm{H}), \delta 7.91(\mathrm{t}, 1 \mathrm{H}), \delta 7.39(\mathrm{~d}, 4 \mathrm{H}), \delta 7.25(\mathrm{~m}, 11 \mathrm{H})$, $\delta 4.36$ (t, $2 \mathrm{H}), \delta 4.28$ (t, $2 \mathrm{H}$ ), $\delta 3.93$ (t, $2 \mathrm{H}$ ), $\delta 3.88$ (s, $3 \mathrm{H}), \delta 3.86$ (t, 2H), $\delta 3,74$ (m, $2 \mathrm{H}$ ), $\delta 3.68(\mathrm{~m}, 2 \mathrm{H}), \delta 3.56(\mathrm{~m}, 6 \mathrm{H}), \delta 3.48(\mathrm{~m}, 2 \mathrm{H}), \delta 3.37(\mathrm{~s}, 3 \mathrm{H}), \delta 3.34(\mathrm{~s}, 3 \mathrm{H}), \delta$ $2.50(\mathrm{t}, 4 \mathrm{H}) ;{ }^{13} \mathrm{C}$ NMR $\left(300 \mathrm{MHz}, \mathrm{CDCl}_{3}\right) \delta 164.87,162.18,144.90,139.05,129.59$, 127.84, 126.64, 112.81, 98.35, 71.81, 70.70, 70.41, 70.34, 69.50, 68.62, 66.57, 41.01, 36.21; ESI MS [M+Na ${ }^{+}$: expected 342.14; observed, 342.2.

3,5-Bis-(2-benzyloxycarbonylamino-acetylamino)-benzoic acid 2-[2-(2-methoxyethoxy)-ethoxy]-ethyl ester (24). 5 (3.0 g, $10 \mathrm{mmol})$ was coupled with Cbz-Gly-OH (2.7 $\mathrm{g}, 12 \mathrm{mmol}$ ) following general procedure (i). Purification was accomplished by chromatography on silica gel by using $\mathrm{DCM} / \mathrm{MeOH}$ as eluent to afford 19 (4.8 g, 7 mmol, 70\%). ${ }^{1} \mathrm{H} \mathrm{NMR}\left(\mathrm{CDCl}_{3}, 400 \mathrm{MHz}\right) \delta 8.88(\mathrm{~s}, 1 \mathrm{H}), \delta 3.37(\mathrm{~s}, 3 \mathrm{H}), \delta 7.91(\mathrm{t}, 1 \mathrm{H})$, $\delta 7.39(\mathrm{~d}, 4 \mathrm{H}), \delta 7.25(\mathrm{~m}, 11 \mathrm{H}), \delta 4.36(\mathrm{t}, 2 \mathrm{H}), \delta 4.28(\mathrm{t}, 2 \mathrm{H}),, \delta 3.93(\mathrm{t}, 2 \mathrm{H}),, \delta 3.88(\mathrm{~s}$, $3 \mathrm{H}), \delta 3.86(\mathrm{t}, 2 \mathrm{H}), \delta 3,74(\mathrm{~m}, 2 \mathrm{H}$ ), $\delta 3.68(\mathrm{~m}, 2 \mathrm{H}), \delta 3.56(\mathrm{~m}, 6 \mathrm{H}), \delta 3.48(\mathrm{~m}, 2 \mathrm{H}), \delta$ $3.37(\mathrm{~s}, 3 \mathrm{H}), \delta 3.34(\mathrm{~s}, 3 \mathrm{H}), \delta 2.50(\mathrm{t}, 4 \mathrm{H}) ;{ }^{13} \mathrm{C}$ NMR $\left(300 \mathrm{MHz}, \mathrm{CDCl}_{3}\right) \delta 166.38$, $152.67,157.85,147.42,140.9,1139.58,131.47,128.71,125.67,126.39,110.54,109.68$,

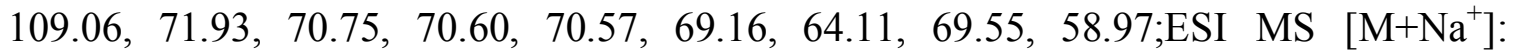
expected 342.14; observed, 342.2 .

3,5-bis-\{2-[2,4-Bis-\{2-[2-(2-methoxy-ethoxy)-ethoxy]-ethoxy\}-5-(2-tritylsulfanylethylcarbamoyl)-benzoylamino]-acetylamino\}-benzoic acid 2-[2-(2-methoxy-ethoxy)ethoxy]-ethyl ester (24). The $\mathrm{Cbz}$ groups of 23 were removed following general procedure (iv) to give the corresponding amine $(0.15 \mathrm{~g}, 0.36 \mathrm{mmol})$, which was coupled with acid $22(0.57 \mathrm{~g}, 0.72 \mathrm{mmol})$ following general procedure $(i)$. Purification was accomplished by chromatography on silica gel by using $\mathrm{DCM} / \mathrm{MeOH}$ as eluent and further purified by reverse phase HPLC to afford $24(0.45 \mathrm{~g}, 0.23 \mathrm{mmol}, 65 \%) .{ }^{1} \mathrm{H}$ NMR $\left(\mathrm{CDCl}_{3}, 400 \mathrm{MHz}\right) \delta 9.96(\mathrm{~b}, 2 \mathrm{H}), \delta 8.97(\mathrm{~s}, 2 \mathrm{H}), \delta 8.85(\mathrm{~b}, 2 \mathrm{H}), \delta 8.60(\mathrm{~s}, 2 \mathrm{H},) \delta 8.10(\mathrm{t}$, $2 \mathrm{H}), \delta 7.38(\mathrm{~d}, 12 \mathrm{H}), \delta 7.26(\mathrm{~m}, 12 \mathrm{H}), \delta 7.18(\mathrm{~m}, 6 \mathrm{H}), \delta 6.58(\mathrm{~s}, 2 \mathrm{H}), \delta 4.48(\mathrm{~m}, 4 \mathrm{H}), \delta$ $4.31(\mathrm{~d}, 4 \mathrm{H}, \mathrm{J}=5.1 \mathrm{~Hz}), \delta 3.82(\mathrm{~m}, 8 \mathrm{H}), \delta 3.68(\mathrm{~m}, 6 \mathrm{H}), \delta 3.51(\mathrm{~m}, 2 \mathrm{H}), \delta 3.32(\mathrm{~m}, 4 \mathrm{H})$, $\delta 2.58(\mathrm{~m}, 6 \mathrm{H}), \delta 2.41(\mathrm{t}, 2 \mathrm{H}), \delta 2.23(\mathrm{t}, 2 \mathrm{H}) . \delta 1.90(\mathrm{~m}, 2 \mathrm{H}) ;{ }^{13} \mathrm{C} \mathrm{NMR}(300 \mathrm{MHz}$, $\left.\mathrm{CDCl}_{3}\right) \delta 166.80,164.87,162.18,147.52,144.90,139.05,131.9,129.59,127.84,126.64$, $112.81,106.88,105.64,98.35,71.81,71.89,70.70,70.69,70.41,70.55,70.34,69.50$, $69.19,68.62,66.57,63.89,58.90,41.01,48.55,36.21$; ESI MS $\left[\mathrm{M}+\mathrm{Na}^{+}\right]$: expected, 1981.8; observed, 1981.8. 


\section{I-6. Synthesis of cross-linked 1-2}

1
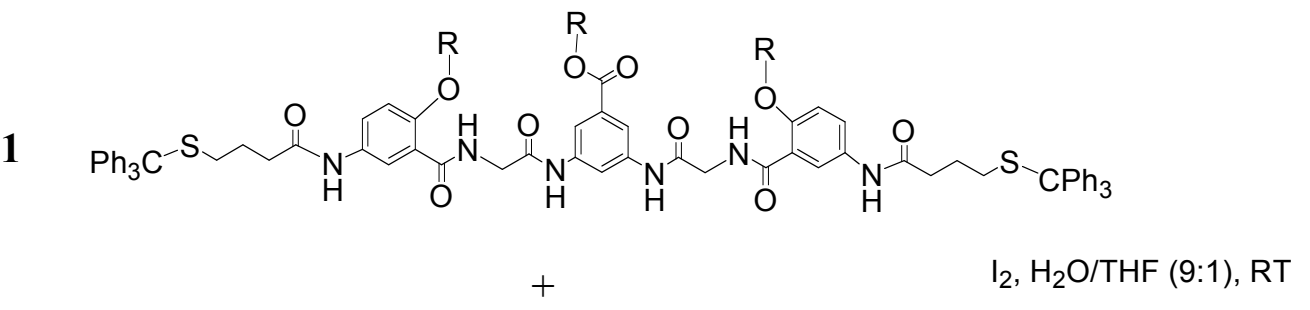

2<smiles>[R]Oc1ccc(NC(=O)CNC(=O)c2cc(NC(=O)CNC(=O)c3cc(O[R])c(C(=O)NCCSCc4ccccc4)cc3O[R])c(O[R])cc2O[R])cc1</smiles><smiles>[R][CH]CCOCCOCCOC</smiles>

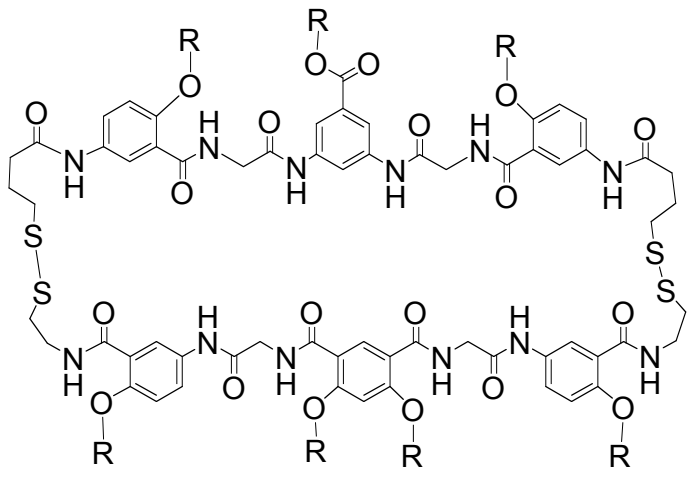

1-2

Crosslinked AB duplex (1-2). 1 (30 mL) and $2(30 \mathrm{~mL})$ were taken from the methylene chloride stock solutions $(2.0 \mathrm{mM})$ separately and mixed in a $250 \mathrm{~mL}$ round bottom flask. Solvent was evaporated in vacco and the residue was dissolved with $120 \mathrm{~mL}$ of iodine solution $(6 \mathrm{mM})$ in mixed solvent (water:THF=9:1). The resulting mixture was stirred at room temperature without inert gas protection. After 10 hours, the reaction mixture was cooled in an ice bath to $0{ }^{0} \mathrm{C}$ and a sodium thiosulfate aqueous solution $(3 \mathrm{mM})$ was added until the color of iodine disappeared. The mixture then was extracted with $\mathrm{CH}_{2} \mathrm{Cl}_{2}$. The organic layer was washed with saturated aqueous $\mathrm{NaCl}$ solution and dried over anhydrous $\mathrm{Na}_{2} \mathrm{SO}_{4}$. Purification was accomplished by chromatography on silica gel by using $\mathrm{DCM} / \mathrm{MeOH}$ as eluent to and further purified by reverse phase HPLC afford 1-2 ( $0.12 \mathrm{~g}, 0.048 \mathrm{mmol}, 80 \%)$ as a white foam. ${ }^{1} \mathrm{H} \mathrm{NMR}\left(\mathrm{CDCl}_{3}, 400 \mathrm{MHz}\right) \delta 9.96(\mathrm{~b}, 2 \mathrm{H})$, $\delta 9.47(\mathrm{~s}, 2 \mathrm{H}), \delta 8.97(\mathrm{~s}, 2 \mathrm{H}), \delta 8.85(\mathrm{~b}, 2 \mathrm{H}), \delta 8.72(\mathrm{~d}, 1 \mathrm{H}), \delta 8.60(\mathrm{~s}, 2 \mathrm{H},) \delta 8.28(\mathrm{~s}, 2$ $\mathrm{H}), \delta 8.10(\mathrm{t}, 2 \mathrm{H}), \delta 8.00(\mathrm{~d}, 2 \mathrm{H}, \mathrm{J}=7.8 \mathrm{~Hz}), \delta 7.01(\mathrm{~m}, 15 \mathrm{H}), \delta 7.38(\mathrm{~d}, 12 \mathrm{H}), \delta 7.26$ $(\mathrm{m}, 12 \mathrm{H}), \delta 7.18(\mathrm{~m}, 6 \mathrm{H}), \delta 6.50(\mathrm{~s}, 1 \mathrm{H}), \delta 6.58(\mathrm{~s}, 2 \mathrm{H}), \delta 4.48(\mathrm{~m}, 4 \mathrm{H}), \delta 4.44(\mathrm{~m}, 2 \mathrm{H})$, $\delta 4.31(\mathrm{~d}, 8 \mathrm{H}, \mathrm{J}=5.1 \mathrm{~Hz}), \delta 4.35(\mathrm{~d}, 2 \mathrm{H}), \delta 3.82(\mathrm{~m}, 8 \mathrm{H}), \delta 3.68(\mathrm{~m}, 9 \mathrm{H}), \delta 3.51(\mathrm{~m}, 8 \mathrm{H})$, $\delta 3.32(\mathrm{~m}, 8 \mathrm{H}), \delta 3.37(\mathrm{~s}, 6 \mathrm{H}), \delta 3.28(\mathrm{~s}, 6 \mathrm{H})$, , $\delta 3.02(\mathrm{t}, 2 \mathrm{H}), \delta 2.70(\mathrm{t}, 2 \mathrm{H}), \delta 2.58(\mathrm{~m}$, $6 \mathrm{H}), \delta 2.41(\mathrm{t}, 2 \mathrm{H}), \delta 2.23(\mathrm{t}, 2 \mathrm{H}) . \delta 2.02(\mathrm{~s}, 3 \mathrm{H}), \delta 1.90(\mathrm{~m}, 2 \mathrm{H}) ;{ }^{13} \mathrm{C} \mathrm{NMR}(300 \mathrm{MHz}$, 
$\left.\mathrm{CDCl}_{3}\right) \delta 179.10, \quad 181.57166 .20,166.80,164.96, \quad 164.87,163.50,162.18,144.90$, $147.52,141.23,139.05,131.91,129.22,128.22,127.84,126.64,121.32,113.85,112.81$, $106.88,105.64,98.35,72.41,71.57,71.15,71.01,70.41,70.34,70.08,, 69.50,69.75$, $59.48,68.62,66.57,59.92,58.86,53.05,41.01,48.61,32.9,31.1,36.21,23.6$; ESI MS $\left[\mathrm{M}+\mathrm{Na}^{+}\right]$: expected, 2481.98; observed, 2481.8.

\section{MALDI TOF Spectra}

\section{II-1. General procedures}
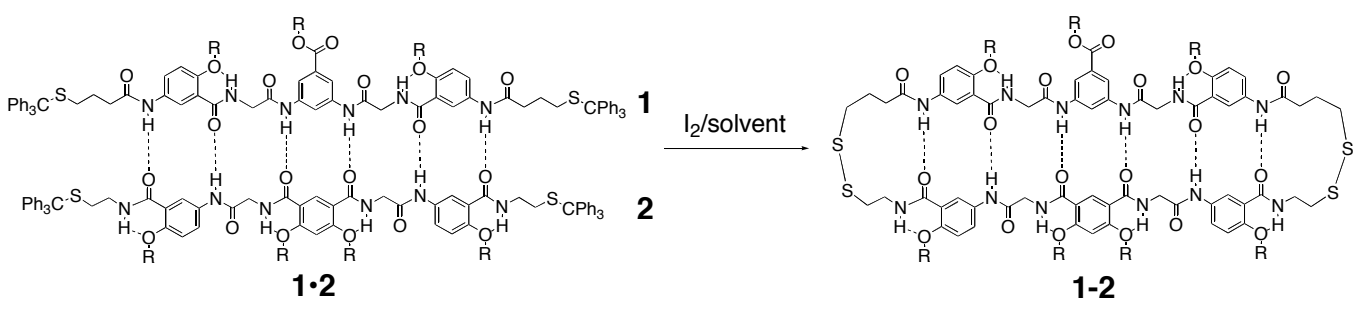

$$
R=\xi \sim 0 \text { ○๐O }
$$
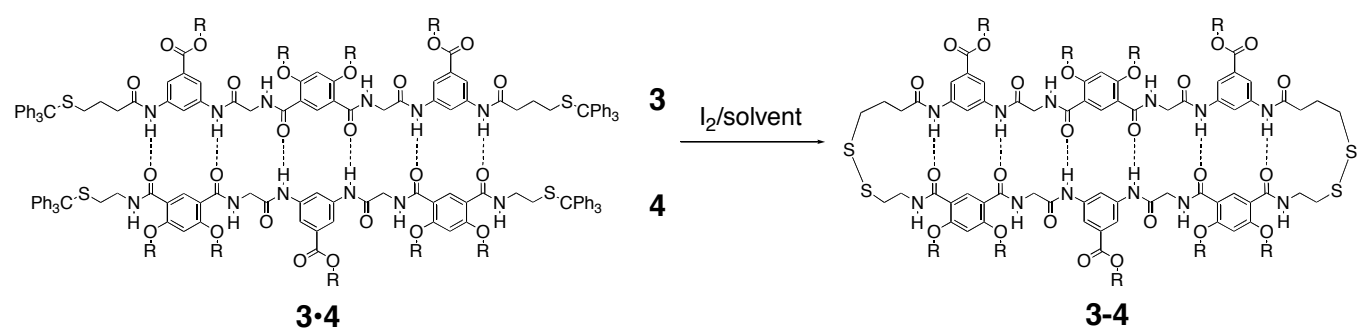

Other possible crosslinked products:
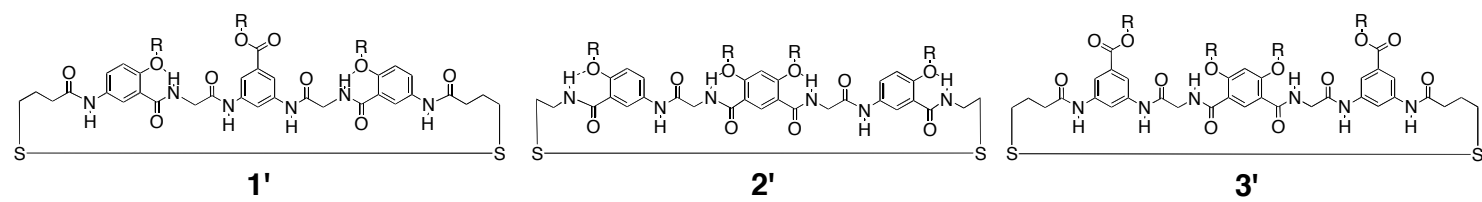

1-1, 2-2, 3-3, 1-3, and 2-3 are the other possible disulifde crosslinked dimers similar to 1-2 and 3-4 but with mismatched $\mathrm{H}$-bonding sites.

General conditions for disulfide crosslinking reactions: A $0.5 \mathrm{~mL}$ portion (or $1 \mathrm{~mL}$ for reactions with only one strand) of each strand was taken from their stock solutions (2.0 $\mathrm{mM}$ in $\mathrm{CH}_{2} \mathrm{Cl}_{2}$ ) separately and mixed in a 2-dram vial. Solvent was evaporated in vacuo and the remaining residue was dissolved in $2 \mathrm{~mL}$ of the corresponding solvent containing $6 \mathrm{mM}$ of iodine. For all the reactions, the final concentration of each oligoamide strand was $0.5 \mathrm{mM}$. The resulting mixture was stirred at room temperature without inert gas protection.

\section{Procedures for MALDI-TOF experiments:}


a. MALDI TOF MS spectra of reaction mixtures were recorded on a Bruker Bifrex IV MS spectrometer.

b. Sampling and Matrix: 2,5-dihydroxybenzoic acid (DHB) in THF was used as the matrix. Two sampling methods, a and $\mathbf{b}$ (see below), were tried for comparison reason. No significant difference was observed in the MS results from these two sampling methods. If there is no specific notice, all the MALDI TOF MS spectra presented are obtained by sampling method a (direct sampling).

Method a: Reaction solution was directly spotted on the layer of the matrix.

Method b: A fraction of $0.5 \mathrm{~mL}$ of a reaction mixture was taken and cooled in an ice bath, to which aqueous sodium thiosulfate $(3 \mathrm{mM})$ was added until the color of iodine disappeared. The mixture then was extracted with $\mathrm{CH}_{2} \mathrm{Cl}_{2}$. The organic layer was washed with saturated $\mathrm{NaCl}$ in $\mathrm{H}_{2} \mathrm{O}$ and dried over anhydrous $\mathrm{Na}_{2} \mathrm{SO}_{4}$, which was then spotted on the layer of the matrix on the target.

II-2. MALDI TOF MS spectra of the crosslinking reaction of the 1:1 mixture of $\mathbf{1}$ and $\mathbf{2}$ in $\mathrm{CH}_{2} \mathrm{Cl}_{2}$.

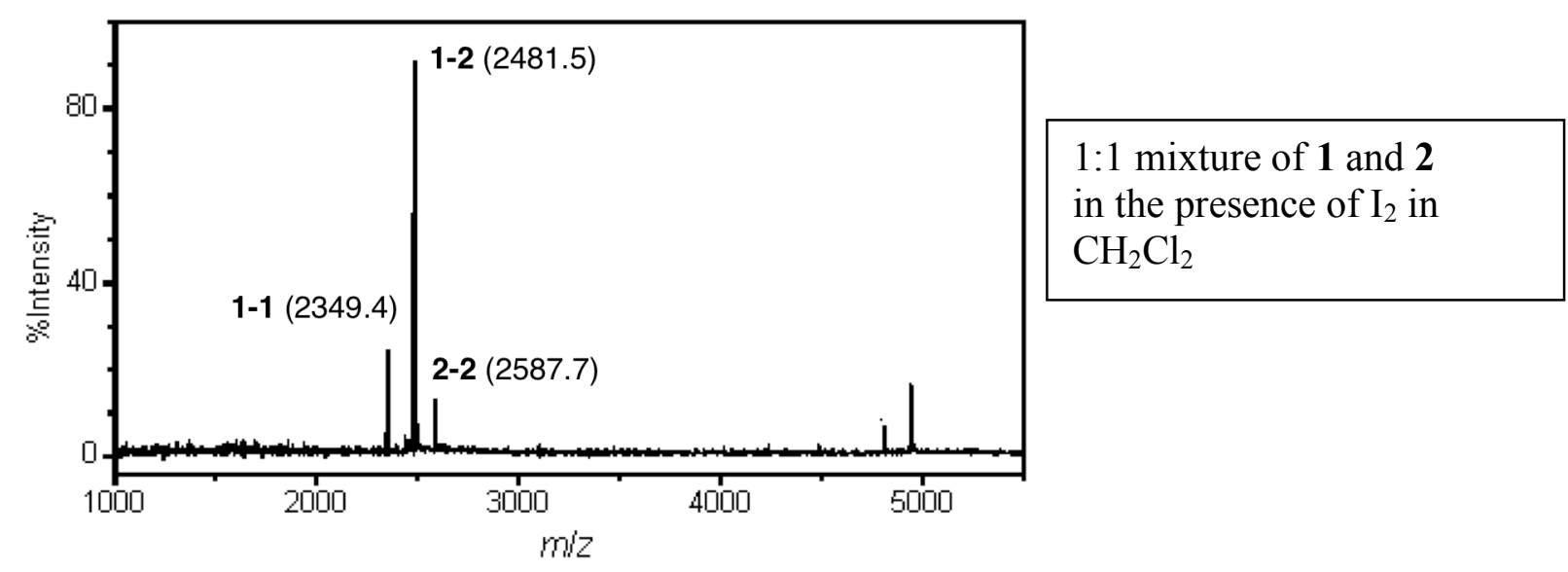

II-3. MALDI TOF MS spectra of the reaction mixture of strands $\mathbf{1}$ and $\mathbf{2}$ in methanol.

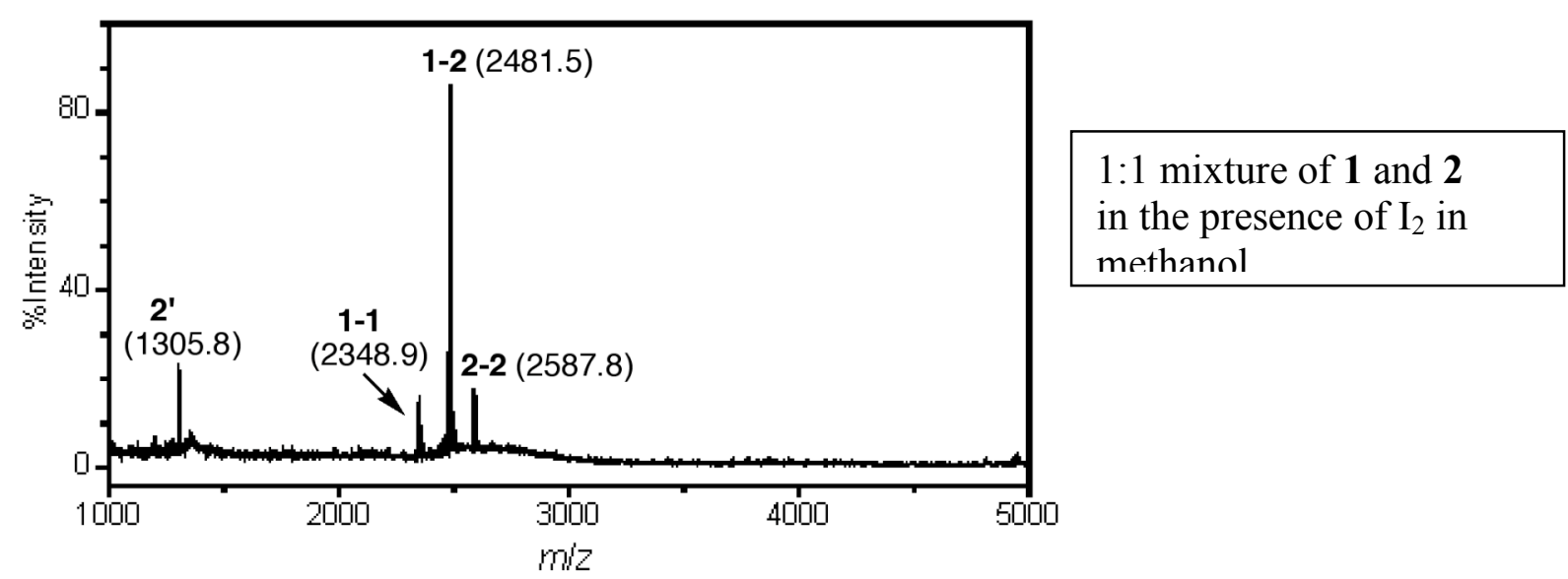


II-4. MALDI-TOF MS spectra of the reaction of 1 alone in water-THF (9:1).

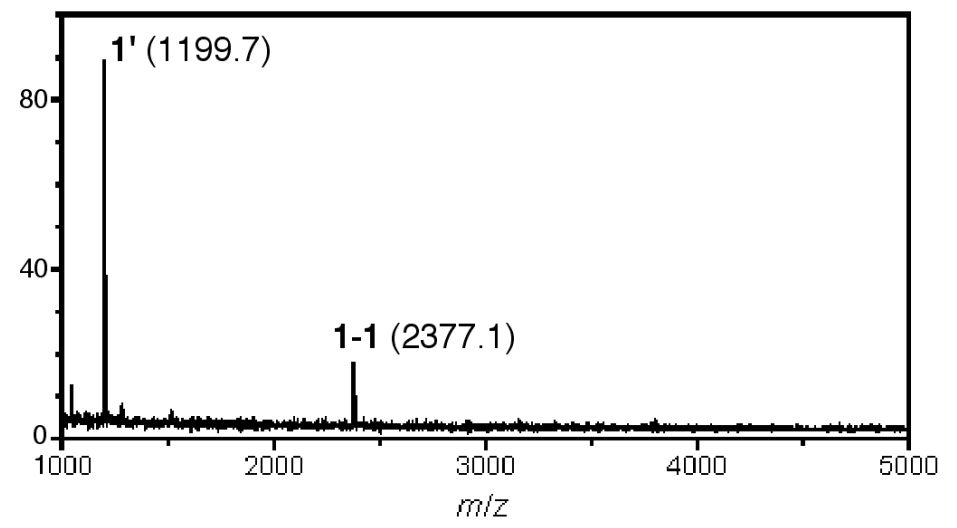

1 alone after 10 hours in water-THF with $\mathrm{I}_{2}(6 \mathrm{mM})$.

II-5. MALDI TOF MS spectra of the reaction of 2 alone in water-THF (9:1).

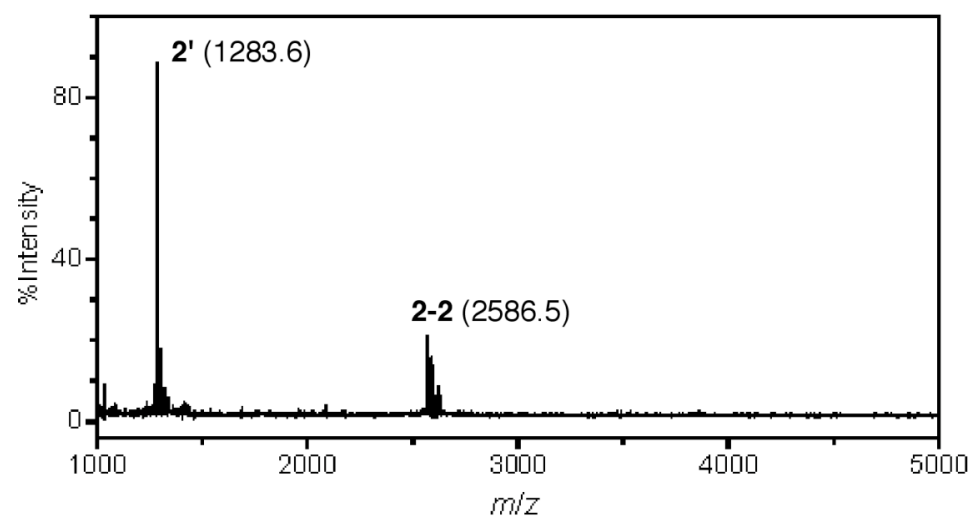

2 alone after 10 hours in water-THF with $\mathrm{I}_{2}(6 \mathrm{mM})$.

II-6. MALDI TOF MS spectra of the crosslinking reaction of 3 alone in water-THF (9:1).

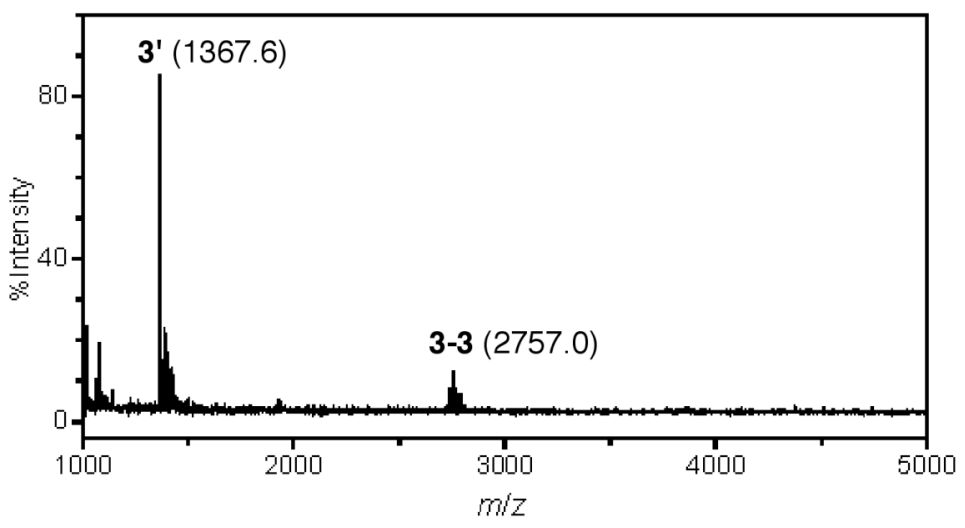

3 alone after 10 hours in water-THF with $\mathrm{I}_{2}(6 \mathrm{mM})$. 
II-7. MALDI-TOF MS spectra of the 1:1 mixture of $\mathbf{1}$ and $\mathbf{3}$ in water-THF (9:1) with $\mathrm{I}_{2}(6 \mathrm{mM})$.

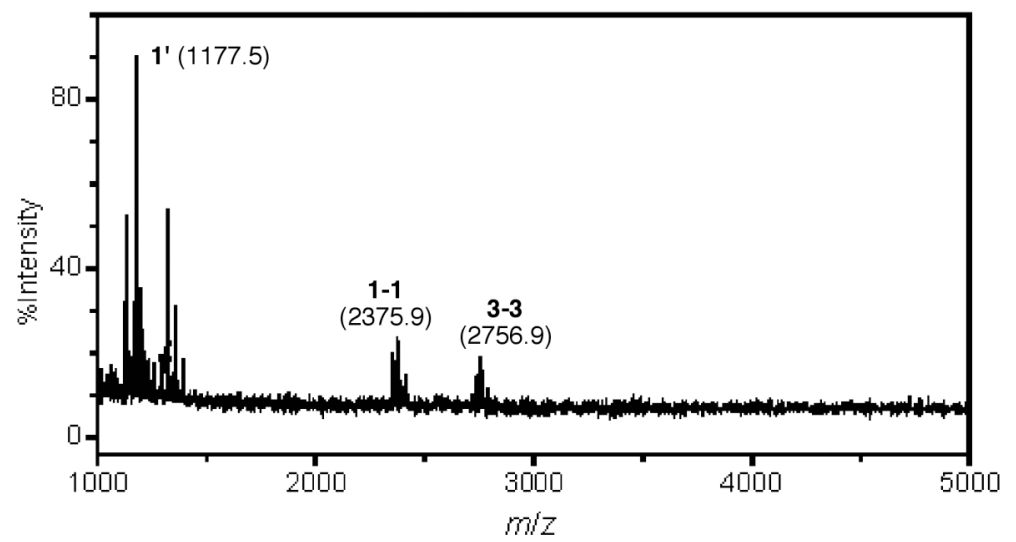

The 1:1 mixture of $\mathbf{1}$ and $\mathbf{3}$ after 10 hours in water-THF with $\mathrm{I}_{2}(6 \mathrm{mM})$.

II-8. MALDI TOF MS spectra of the 1:1 mixture of 2 and 3 in water-THF (9:1) with $\mathrm{I}_{2}(6 \mathrm{mM})$.

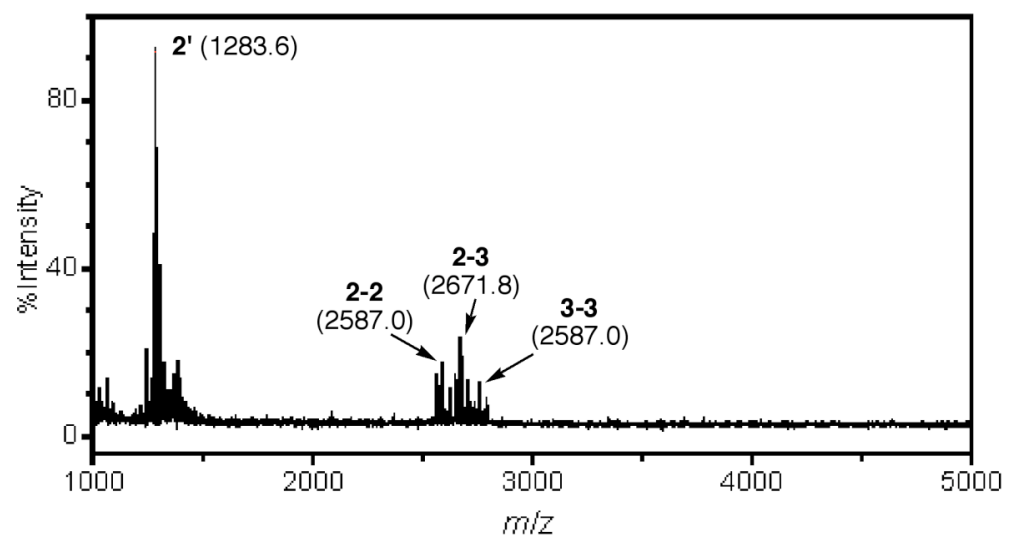

The 1:1 mixture of $\mathbf{2}$ and $\mathbf{3}$ only after 10 hours in waterTHF with $\mathrm{I}_{2}(6 \mathrm{mM})$.

II-9. MALDI-TOF MS spectra of the 1:1 mixture of 3 and 4 in water-THF $(9: 1)$ with $\mathrm{I}_{2}(6 \mathrm{mM})$.

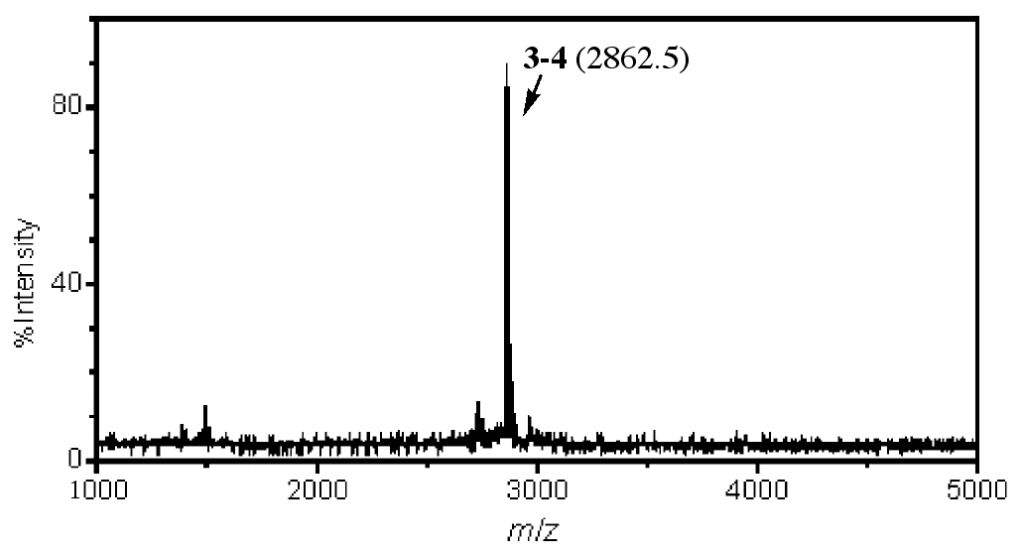




\section{Traces from Reverse-Phase HPLC Experiments}

The HPLC characterization was carried out on a Shimadzu 10AV VP. All traces were recorded with a UV detector at $254 \mathrm{~nm}$. The column was a ZORBAX Eclipse XDB-C8 (4.6 x $150 \mathrm{~mm}, 5 \mu \mathrm{m})$ of Agilent Technologies. A $5 \mu \mathrm{L}$ aliquot of a given reaction mixture solution was directly injected for HPLC analysis at ambient temperature. HPLC conditions are: $1.0 \mathrm{~mL} / \mathrm{min}$; methanol-water gradient elution; methanol $40 \%$ to $65 \%$ in 20 minutes followed by methanol $65 \%$ to $80 \%$ in 25 minutes. Fraction of eluent corresponding to each peak was collected and analyzed by MALDI TOF mass spectroscopy for peak identification.

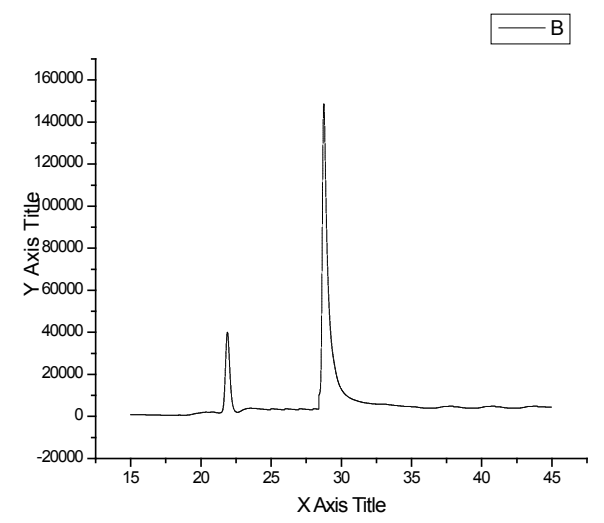

1 alone $+\mathrm{I}_{2}$

The 1st peak ( $\mathrm{rt}=22 \mathrm{~min})$ : homo-dimer 1-1 the $2^{\text {nd }}$ peak $(\mathrm{rt}=29 \mathrm{~min})$ : cyclic-monomer $\mathbf{1}^{\text {' }}$

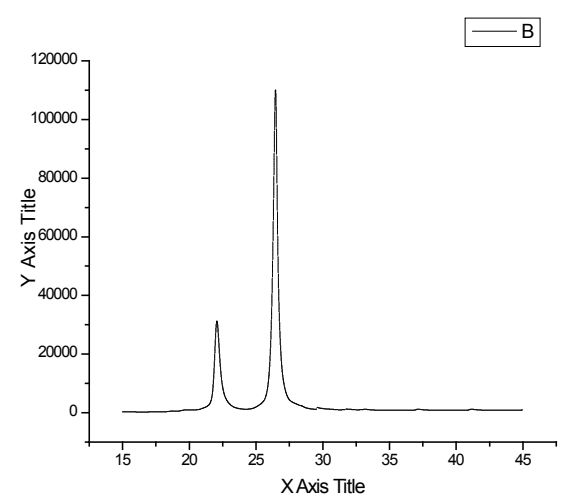

2 alone $+\mathrm{I}_{2}$

The 1st peak ( $\mathrm{rt}=22 \mathrm{~min})$ : homo-dimer $\mathbf{2 - 2}$ the $2^{\text {nd }}$ peak ( $\left.\mathrm{rt}=26 \mathrm{~min}\right)$ : cyclic-monomer 2 '

\section{Reference:}

1. Gong, B.; Yan, Y.; Zeng, H. J. Am. Chem. Soc. 1999, 121, 5607. 